\title{
O impacto na saúde mental de universitários de Alagoas decorrente da pandemia do COVID-19
}

\author{
The impact on mental health in university students in \\ Alagoas during the COVID-19 pandemic \\ Vanina Papini Góes Teixeira ${ }^{1 *}$, Luiz Raphael de Melo Tavares², \\ Sheilla Alencar Bezerra Ferraz Barbosa ${ }^{2}$
}

\begin{abstract}
RESUMO
INTRODUÇÃO: A prevalência de ansiedade e depressão em universitários é alta, devido às exigências aos quais são submetidos. O contexto da pandemia de COVID-19 se apresenta como um fator de risco para o desenvolvimento destes transtornos. OBJETIVO: verificar o impacto na saúde mental de universitários de Alagoas devido ao momento de pandemia do COVID-19, e identificar a relação entre a prática de atividade física e sintomas de ansiedade e depressão. MÉTODO: estudo observacional, transversal. Utilizou-se um questionário sociodemográfico e Inventários de Ansiedade e Depressão de Beck, com amostra não probabilística, composta de 75 universitários, de instituições públicas e privadas de Alagoas. RESULTADOS: idade média dos sujeitos da amostra foi de 25,54 anos $( \pm 10,2)$, com a media de 170 dias sem aula ou em aula remota $( \pm 68,7)$; média de $13,6( \pm 9,9)$ para depressão e média de $15,5( \pm 13,2)$ para ansiedade; correlação moderada negativa entre prática de atividade física e depressão $(r=-0.509, p \leq 0.05)$ e ansiedade $(r=-0.565, p$ $\leq 0.05$ ), indicando que quanto menor é o tempo de prática de atividade física mais grave é o nível depressão, e e mais grave a ansiedade. A literatura aponta que os universitários foram um dos grupos mais afetados psicologicamente pela pandemia de COVID-19. Pode-se afirmar que, na amostra, quanto menor foi o tempo de atividade física, maior foi o nível de ansiedade e depressão.
\end{abstract}

Palavras-chave: Ansiedade; Depressão; Pandemia; Atividade Física; Universitários.

\section{ABSTRACT}

INTRODUCTION: The prevalence of anxiety and depression in university students is high, due to the demands to which they are submitted. The context of the COVID-19 pandemic presents itself as a risk factor for the development of these disorders. OBJECTIVE: to verify the impact on the mental health of universities in Alagoas due to the moment of the COVID-19 pandemic, and to identify the relationship between the practice of physical activity and symptoms of anxiety and depression. METHOD: a cross-sectional study. A sociodemographic questionnaire and Beck Anxiety and Depression Inventories were used, with a non-probabilistic sample, consisting of 75 university students from public and private institutions in Alagoas. RESULTS: mean age of the sample was 25.54 years $( \pm 10.2)$, with an average of 170 days without class or in remote classes $( \pm 68.7)$; mean of $13.6( \pm 9.9)$ for depression and mean of $15.5( \pm 13.2)$ for anxiety; moderate negative correlation between physical activity practice and depression $(r=-0.509, p<0.05)$ and anxiety $(r=-0.565, p<$ 0.05 ), indicating that the shorter the time of physical activity practice, the more severe the level depression and anxiety. The literature indicates that university students were one of the groups most psychologically affected by the COVID-19

\footnotetext{
${ }^{1}$ Centro Universitário Cesmac *Vanina.papini@ cesmac.edu.br

${ }^{2}$ Centro Universitário Cesmac
} 
pandemic. It can be said that, in the sample, the shorter the time of physical activity, the higher the level of anxiety and depression.

Keywords: Anxiety; Depression; Pandemic; Physical Activity; University Sutudents.

\section{INTRODUÇÃO}

De acordo com a World Health Organization (2017), o Brasil é o $5^{\circ}$ país com o maior número de casos de depressão, acometendo 5,8\% da população brasileira, e o país com o maior número de casos de ansiedade, tendo $9,3 \%$ da população afetada por esse transtorno.

Um dos grupos mais afetados por esses transtornos é o de estudantes universitários. Fernandes et al (2018) falam sobre uma alta prevalência de depressão em estudantes universitários, tendo uma média de 30,6\%, e ainda apontam que a prevalência de ansiedade, nessa mesma população, de $63 \%$ a $92 \%$.

Segundo Silva, Panosso e Donadon (2018), mudanças físicas, sociais, psicológicas e estressores no ambiente acadêmico, são responsáveis por afetar a saúde mental dessa população. Os estudantes estão passando por um período de transição fisiológica, psicológica e social, saindo da adolescência e entrando na fase adulta, além disso, a vida acadêmica traz novas responsabilidades para esse grupo.

Em 11 de março de 2020, a Organização Mundial da Saúde declarou estado de pandemia do COVID-19 (WORLD HEALTH ORGANIZATION, 2020). Com isso, os países tiveram que tomar providências para impedir a contaminação. Zwielewski et al. (2020) relatam que, apesar de ser considerada extrema, a quarentena foi uma das principais medidas implantadas por ser eficaz contra a disseminação do vírus.

Segundo Brooks et al. (2020), estudos feitos com pessoas que passaram por quarentena, mostraram que estas apresentavam: perturbação emocional, depressão, estresse, irritabilidade, insônia, sintomas de estresse pós-traumático, raiva e exaustão emocional. Não só isso, mas os autores também colocam que quanto maior o tempo de quarentena, mais elevado os sintomas se apresentavam.

Pensando nisso, o presente artigo tem como objetivo verificar o impacto na saúde mental de universitários de Alagoas em decorrência da pandemia de COVID-19 além de 
identificar a relação entre prática de atividade física e sintomas de ansiedade e depressão durante o isolamento social.

\section{MÉTODO}

Trata-se de um estudo observacional, analítico, transversal. O estudo foi conduzido por meio eletrônico, com estudantes universitários de instituições de ensino superior, públicas e privadas, do estado de Alagoas.

Os estudantes foram contactados através de e-mail, WhatsApp, Instagram, ou qualquer outro meio eletrônico, e foram informados sobre a pesquisa, sendo convidados a participar.

Aqueles que concordaram com a participação assinaram o Termo de Consentimento Livre e Esclarecido - TCLE, baseado nas diretrizes da resolução CNS/MS 466/12 e CNS/MS 510/16.

Após o aceite e assinatura do TCLE, responderam os instrumentos, que foram disponibilizados por meio eletrônico.

Foi utilizado um questionário sociodemográfico, com as características tais como, nome, sexo, idade, estado civil, curso superior e instituição de ensino; como também abordou as questões relacionadas a diagnósticos de transtornos psiquiátricos prévios a pandemia do COVID-19 e a questões relacionadas a prática de atividade física durante o período de isolamento social, sendo classificada em comportamento sedentário (CS) quando não praticou atividade física durante o isolamento social, baixa atividade física (BAF) quando praticou menos que uma hora de atividade física semanal, moderada atividade física (MAF) durante o isolamento social quando praticou entre uma e duas horas de atividade física durante a semana, e intensa atividade física (IAF) quando praticou mais que duas horas de atividade física semanal, durante o isolamento social.

Para a avaliação dos sintomas de depressão foi utilizado o Inventário de Depressão de Beck (BDI), que é uma escala autoaplicável, e fornece uma avaliação quantitativa, presença e intensidade, dos sintomas depressivos.

De acordo com Cunha (2001) o inventário foi originalmente criado por Beck, Ward, Mendelson, Mock e Erbaugh em 1961 e posteriormente revisado por Beck, Rush, Shaw e Emery em 1982. A escala consiste em 21 itens, incluindo sintomas e atitudes, cuja intensidade varia de 0 a 3 (BECK; STEER, 1993). O Inventário de Depressão de Beck foi traduzido e adaptado para o Brasil por Cunha (2001). Tem os pontos de corte: pontuação 
de 0 a 13, sintomas mínimos de depressão; de 14 a 19, sintomas leves de depressão; de 20 a 28, sintomas moderados; e, de 29 a 63, sintomas graves de depressão (GORESTEIN; WANG, 2016).

Para a avaliação de níveis de sintomas de ansiedade foi utilizado o Inventário de Ansiedade de Beck - BAI. Esse inventário foi originalmente desenvolvido por Beck, Epstein, Brown e Steer, em 1988 e adaptado para o Brasil por Cunha (2001).

O BAI é composto por 21 itens que apresentam informações descritivas dos sintomas de ansiedade. Esses devem ser avaliados pelo sujeito em referência a si mesmo em razão da gravidade e frequência de cada item numa escala de 0 a 3 pontos. Para a mensuração do nível de sintomas de ansiedade é adotado o seguinte: pontuação de 0 a 10, nível mínimo de sintomas de ansiedade; de 11 a 19 pontos, nível leve de ansiedade; de 20 a 30 pontos, nível moderado; e de 31 a 63 pontos, nível grave de sintomas de ansiedade (CUNHA, 2001).

A análise estatística foi realizada utilizando o Statistical Package for the Social Sciences (SPSS) para Windows (Inc., Chicago, Illinois, EUA) software versão v.21.0.

Procedeu-se, inicialmente, à estatística descritiva. Para tanto, foram calculadas as medidas-resumo e construídas tabelas adequadas à natureza e ao nível de mensuração das variáveis envolvidas.

Foi utilizado de coeficiente de correlação de Pearson $(r)$ para verificar a associação entre níveis de depressão e níveis de ansiedade com idade e sexo, como também com a prática de atividade física. A margem de erro assumida foi de 5\%.

Este protocolo de pesquisa foi submetido à avaliação do Comitê de Ética em Pesquisa do Centro Universitário Cesmac, CAAE 34596620.8.0000.0039.

\section{RESULTADOS}

Os dados referentes às características da amostra, no que se refere a dados sociodemográficos tais como idade, gênero, etnia, estado civil, área de curso superior, tempo sem aula ou em aula remota, prática de atividade física e autorrelato de depressão e ansiedade estão disponibilizados nas tabelas 1 e 2 . 
Tabela 1. Características sociodemográficas da amostra. $\mathrm{N}=75$.

\begin{tabular}{lcccc}
\hline Variáveis & M & DP & $\begin{array}{c}\text { Erro } \\
\text { Padrão }\end{array}$ & Min-Máx \\
\hline Idade (anos) & 25.54 & 10.20 & 1,20 & $18-63$ \\
$\begin{array}{l}\text { Dias sem aula ou } \\
\text { com aula remota }\end{array}$ & 170 & 68.7 & 8.2 & $0-279$ \\
\hline
\end{tabular}

Fonte: Teixeira, Tavares, Barbosa (2021).

Participaram deste estudo 75 universitário do Estado de Alagoas. A idade média dos sujeitos que compõem a amostra foi de 25,54 anos $( \pm 10,2)$, e apresentam, em média, 170 dias sem aula ou em aula remota $( \pm 68,7)$, conforme disposto na tabela 1 .

Pode-se observar na tabela 2, que 66,7\% (n=53) dos indivíduos da amostra são do gênero feminino e 33,3\% $(\mathrm{n}=22)$ do gênero masculino. No que se refere à etnia, na qual os próprios sujeitos se avaliavam, tem-se: $60,6 \%(n=40)$ se considera branca, $25,8 \%$ $(n=17)$ se considera negra, $18,1 \%(n=12)$ se considera parda e $9 \%$ se diz de outra etnia. Em relação ao estado civil tem-se $80 \%(\mathrm{n}=60)$ de solteiros, $10,7 \%(\mathrm{n}=8)$ de indivíduos casados (ou união estável), 5,3\% $(n=4)$ de viúvos e $4 \%(n=3)$ de pessoas divorciadas.

Tabela 2. Gênero, Etnia e Estado Civil. N=75.

\begin{tabular}{lccc}
\hline \multicolumn{1}{c}{ Variáveis } & Categorias & N & \% \\
\hline Gênero & & & \\
& Feminino & 53 & 66,7 \\
\multirow{2}{*}{ Etnia } & Masculino & 22 & 33,3 \\
& & & \\
& Branca & 40 & 60,6 \\
& Negra & 17 & 25,8 \\
& Parda & 12 & 18,1 \\
\multirow{3}{*}{ Estado Civil } & Outras & 6 & 9,0 \\
& & & \\
& Solteiro (a) & 38 & 65,5 \\
& Casado (a) & 09 & 15,5 \\
& Divorciado (a) & 05 & 8,6 \\
& Viúvo (a) & 06 & 10,3 \\
\hline
\end{tabular}

Fonte: Teixeira, Tavares, Barbosa (2021).

Em relação as áreas do conhecimento, os estudantes estão distribuídos em quatro áreas, sendo: 61,3\% ( $\mathrm{n}=46)$ na Saúde, $20 \%(\mathrm{n}=15)$ em Humanas, 14,7\% ( $\mathrm{n}=11)$ em Exatas, $2,7 \%(\mathrm{n}=2)$ nas Sociais e 1,3\% $(\mathrm{n}=1)$ não respondem, como está disposto na tabela 3. No que se refere a autorrelato de ansiedade e/ou depressão, os resultados encontrados 
foram que $72 \%(n=54)$ referem não ter sintomas e $28 \%(n=21)$ referem ter sintomas de ansiedade e/ou depressão (tabela 3).

Tabela 3. Áreas de conhecimento e autorrelato de ansiedade e/ou depressão. N=75.

\begin{tabular}{lccc}
\hline Variáveis & Categorias & $\mathbf{N}$ & \% \\
\hline Áreas do conhecimento & & & \\
& Saúde & 46 & 61,3 \\
& Humanas & 15 & 20,0 \\
& Exatas & 11 & 14,7 \\
& Sociais & 2 & 2,7 \\
& Não respondeu & 1 & 1,3 \\
Autorrelato de ansiedade & & & \\
e/ou depressão & Não & 54 & 72,0 \\
& Sim & 21 & 28,0 \\
\hline
\end{tabular}

Fonte: Teixeira, Tavares, Barbosa (2021).

Com relação à prática de atividade de atividade física durante o período de isolamento social foram encontrados os seguintes resultados: $29,3 \%(n=22)$ apresentaram um comportamento sedentário (CS) nesse período, 26,7\% ( $\mathrm{n}=20)$, praticaram atividade física de forma intensa (IAF), $24 \%(n=18)$ tiveram uma prática baixa de atividade física (BAF) no período de isolamento social e $20 \%(\mathrm{n}=15)$ tiveram uma prática moderada de atividade física (MAF) durante esse período (tabela 4).

Tabela 4. Prática de atividade física durante o isolamento social. $\mathrm{N}=75$.

\begin{tabular}{llll}
\hline \multicolumn{1}{c}{ Variáveis } & Categorias & N & $\%$ \\
\hline $\begin{array}{l}\text { Prática de atividade física } \\
\text { durante o isolamento social }\end{array}$ & & & \\
& CS & 46 & 61,3 \\
& IAF & 15 & 20,0 \\
& BAF & 11 & 14,7 \\
& MAF & 2 & 2,7 \\
\hline CS: comportamento sedentário; IAF: intensa atividade física; BAF: baixa atividade \\
$\begin{array}{c}\text { física; MAF: moderada atividade física. } \\
\text { Fonte: Teixeira, Tavares, Barbosa (2021). }\end{array}$
\end{tabular}


Em relação aos níveis de gravidade de depressão e ansiedade a amostra desse estudo apresentou uma média de 13,6 ( $\pm 9,9)$ para depressão e uma média de 15,5 $( \pm 13,2)$ para ansiedade, apontando que, de acordo com as normas dos instrumentos utilizados, a amostra apresenta níveis leves de depressão e ansiedade (tabela 5).

Tabela 5. Depressão e ansiedade durante o isolamento social. $\mathrm{N}=75$.

\begin{tabular}{ccccc}
\hline Variáveis & M & DP & $\begin{array}{c}\text { Erro } \\
\text { Padrão }\end{array}$ & Min-Máx \\
\hline Depressão (BDI) & 13,6 & 9,9 & 1,1 & $0-39$ \\
Ansiedade (BAI) & 15,5 & 13,2 & 1,5 & $0-53$ \\
\hline
\end{tabular}

BDI: Inventário de depressão de Beck; BAI: Inventário de Ansiedade de Beck. Fonte: Teixeira, Tavares, Barbosa (2021).

Na Tabela 6 estão as informações relativas à prática de atividade física durante o período de isolamento social e sua relação com a depressão e a ansiedade, para tanto foi utilizado o método de correlação bivariada de Pearson ( $r$ ).

Foi encontrada uma correlação moderada negativa entre prática de atividade física e depressão ( $r=-0.509, p \leq 0.05)$, indicando que quanto menor é o tempo de prática de atividade física mais grave é o nível depressão, e também encontrou-se correlação moderada negativa entre prática de atividade física e ansiedade $(r=-0.565, p \leq 0.05)$, revelando que quanto menor o tempo de prática de atividade física mais grave a ansiedade. Além disso, encontrou-se correlação forte e positiva entre ansiedade e depressão $(r=0.729, p \leq 0.001)$, indicando que quanto mais grave a depressão, mais grave a ansiedade (tabela 6).

Tabela 6. Correlação entre tempo de prática de atividade física durante o isolamento social e depressão e ansiedade ( $\mathrm{r}$ de Pearson).

\begin{tabular}{|c|c|c|}
\hline & Depressão (BDI) & Ansiedade (BAI) \\
\hline $\begin{array}{l}\text { Prática de Atividade Física } \\
\text { durante o isolamento social }\end{array}$ &,$- 509 *$ &,$- 565^{*}$ \\
\hline Depressão (BDI & &, $792 * *$ \\
\hline BDI: Inve & $\begin{array}{l}\text { eck; BAI: Inventá } \\
k * p \leq 0,01 \\
* p \leq 0,05\end{array}$ & Beck. \\
\hline
\end{tabular}


Fonte: Teixeira, Tavares, Barbosa (2021).

\section{DISCUSSÃO}

As demandas da rotina acadêmica, por si só, requerem do estudante universitário um repertório de recursos cognitivos e emocionais. Ao adentrar o ensino superior, o acadêmico se vê em um ambiente potencialmente estressor, cenário este que pode ter relação com a alta prevalência de problemas de natureza psicoafetiva. Tais problemas, quando combinados ao volume de exigências da academia, tendem a ser desvalorizados pelo corpo discente, levando a uma identificação tardia de sintomas de depressão e ansiedade. (PADOVANI et al, 2014)

Estudos apontam que os estudantes universitários podem apresentar valores de prevalência de depressão até três vezes maior do que a população não universitária. Essa discrepância é ainda maior em transtornos de ansiedade, dada a estimativa de que entre $63 \%$ e $92 \%$ da população universitária apresenta sintomas de ansiedade. (FERNANDES et al, 2018)

Em situações normais, isso já poderia ser considerado como um grave problema de saúde pública. Mas, como Belasco e Fonseca (2020) ressaltam, o bem-estar psicológico da população pode ainda ser afetado em um contexto epidemiológico no qual as pessoas têm medo de contrair uma doença potencialmente letal.

A rápida disseminação de COVID-19 pelo mundo fez a Organização Mundial da Saúde classificar a doença como uma pandemia. Desde então, muitos países têm adotado medidas a fim de reduzir o pico de incidência, tais como a implementação de distanciamento social em estabelecimentos, fechamento de escolas e universidades e quarentena para a população. (BELASCO; FONSECA, 2020).

Neste sentido, a literatura aponta que estudantes universitários foram um dos grupos mais afetados psicologicamente pela pandemia de COVID-19. Entre as principais causas, destacam-se aquelas associadas à implementação da quarentena: quebra da rotina acadêmica, afastamento de amigos e colegas, preocupação com o atraso de atividades, interrupção de aulas práticas e estágios e perdas de aprendizagem. (GUNDIM et al, 2021)

Em nossa pesquisa, contudo, a amostra apresentou níveis leves de depressão e ansiedade, o que não corresponde aos achados de outros estudos sobre o tema. Em pesquisa realizada no estágio inicial da pandemia, na China, foi constatado que $28,8 \%$, 
$16,5 \%$ e $8,1 \%$ dos respondentes apresentaram, respectivamente, sintomas de moderados a graves de ansiedade, depressão e estresse (WANG et al 2020). Essa discrepância se justifica pela técnica de amostragem utilizada, configurando uma limitação do nosso estudo.

No que diz respeito à correlação entre o tempo de prática de atividade física e o nível de depressão e ansiedade, os resultados são semelhantes aos achados em estudos antecedentes. Em pesquisa recente, foi constatado que os níveis de atividade física de homens e mulheres diminuíram em decorrência das medidas de restrição utilizadas para mitigar a pandemia. Entre aqueles que conseguiram manter os níveis de atividade física que tinham no período pré-pandemia, os níveis dos sintomas de ansiedade e depressão foram consideravelmente mais leves. (PUCCINELLI et al, 2021)

Deste modo, os achados apontam para a necessidade de reforçar a importância da atividade física nesse contexto, como uma estratégia de enfrentamento ao sofrimento psíquico causado pela quarentena imposta aos estudantes universitários. Além disso, outra estratégia interessante seria viabilizar um espaço de escuta e intervenção terapêutica dentro das instituições, a fim de identificar problemas de natureza psicoafetiva e oferecer ações de promoção e prevenção à saúde mental dos discentes.

\section{CONCLUSÃO}

Essa pesquisa verificou o impacto causado pela pandemia na saúde mental de estudantes universitários de Alagoas. As medidas implementadas para conter a disseminação do vírus, como a quarentena, podem afetar a saúde mental dos discentes. Mensurando os níveis de ansiedade e depressão, e correlacionando esses níveis com os dados coletados dessa população, foi possível identificar a presença de sintomas leves desses transtornos. Verificou-se também a correlação entre o nível de atividade física e a prevalência desses sintomas, de modo que é possível afirmar que quanto menor for o tempo de atividade física, maior será o nível de ansiedade e depressão da população-alvo.

Em situações de emergência no âmbito da saúde pública, o combate ao agente patogênico e a consequente preservação da saúde física das pessoas recebe a atenção de gestores e profissionais de saúde, enquanto as políticas de saúde mental ficam em segundo plano. Entretanto, as implicações psicológicas da pandemia não podem ser descartadas, tendo em vista que os problemas psíquicos decorrentes podem ser mais prevalentes e 
duradouros. Isto se mostra verdadeiro no caso dos estudantes universitários, que se constituem como pessoas suscetíveis ao sofrimento psíquico em decorrência da rotina acadêmica a qual estão submetidos, sobretudo levando em conta as mudanças causadas pelas medidas de restrição adotadas para combater a disseminação do COVID-19.

\section{REFERÊNCIAS}

BECK, A. T; STEER, R. A. Beck Depression Inventory. Manual. San Antonio: Psychology Corporation, 1993.

BELASCO, A. G. S.; FONSECA, C. D. Coronavírus 2020. Revista Brasileira de Enfermagem, Brasília, v.73, n.2, e2020n2, 2020. Disponível em: <http://dx.doi.org/10.1590/0034-7167-2020730201>. Acessado em: 18 de junho de 2021.

BROOKS, S. K. et al. The psychological impact of quarantine and how to reduce it: rapid review of the evidence. The Lancet, Londres, v. 395, p. 912-20, 14 mar. 2020. Disponível em: <https://doi.org/10.1016/ S0140-6736(20)30460-8>. Acesso em: 18 de junho de 2021.

CUNHA, J. A. Manual da versão em português das Escalas Beck. São Paulo: Casa do Psicólogo, 2001.

FERNANDES, M. A. et al. Prevalência de sintomas ansiosos e depressivos em universitários de uma instituição pública. Revista Brasileira de Enfermagem, Brasília, v.71, supl. 5, 2018. Disponível em: <https://doi.org/10.1590/0034-7167-2017-0752>. Acessado em: 19 de junho de 2021.

GORESTEIN, C.; WANG, Y.P.; HUNGERBÜHLER, I. (orgs). Instrumentos de avaliação em saúde mental. Porto Alegre: Artmed, 2016.

GUNDIM, V. A. et al. Saúde mental de estudantes universitários durante a pandemia de COVID-19. Rev baiana enferm. 2021;35:e37293. Disponível em: < https://periodicos.ufba.br/index.php/enfermagem/article/view/37293>. Acessado em: 17 de junho de 2021.

PADOVANI, R. C. et al. Vulnerabilidade e bem-estar psicológicos do estudante universitário. Revista Brasileira de Terapias Cognitivas, Rio de Janeiro, v.10, n.1, jun. 2014. Disponível em: <http://dx.doi.org/10.5935/1808-5687.20140002 >. Acessado em: 2 de julho de 2020.

PUCCINELLI, P.J et al. Reduced level of physical activity during COVID-19 pandemic is associated with depression and anxiety levels: an internet-based survey. BMC Public 
Health 21, 425 (2021). Disponível em: 〈https://doi.org/10.1186/s12889-021-10470-z〉. Acessado em: 17 de junho de 2021.

SILVA, D. R. DA; PANOSSO, I. R.; DONADON, M. F. revisão crítica da literatura Ansiedade em universitários: fatores de risco associados e intervenções - uma revisão crítica da literatura. Psicologia - Saberes \& Práticas, v. 1, n. 2, p. 1-10, 2018.

WANG, C. et al. Immediate psychological responses and associated factors during the initial stage of the 2019 coronavirus disease (COVID-19) epidemic among the general population in China. International Journal of Environmental Research and Public Health, v. 17, n. 5, 2020. Disponível em: 〈http://dx.doi.org/10.3390/ijerph17051729>. Acesso em: 21 de maio de 2021.

WORLD HEALTH ORGANIZATION. Director-General's opening remarks at the media briefing on COVID-19-11 March 2020. WHO, 2020. Disponível em: $<$ https://www.who.int/director-general/speeches/detail/who-director-general-sopening-remarks-at-the-media-briefing-on-covid-19---11-march-2020>. Acesso em: 18 de junho de 2021.

WORLD HEALTH ORGANIZATION. Depression and Other Common Mental Disorders: Global Health Estimates. Geneva, 2017. Disponível em:

<https://apps.who.int/iris/bitstream/handle/10665/254610/WHO-MSD-MER-2017.2eng.pdf? sequence=1>. Acesso em: 18 de junho de 2021 .

ZWIELEWSKI, G. et al. Protocolos para tratamento psicológico em pandemias: as demandas em saúde mental produzidas pela COVID-19. Revista debates in psychiatry, v. 3, p. 2-9, 2020. 